\title{
Vitamin D supplementation in multiple sclerosis patients in 2012: hype or reality as an adjunctive therapy?
}

\author{
V. van Pesch • C. J. M. Sindic
}

Published online: 16 November 2012

(C) Belgian Neurological Society 2012

Based on epidemiological case-control studies, vitamin D deficiency is now recognized as an independent environmental risk factor for developing multiple sclerosis (MS). Studies indicate that vitamin D, besides regulating bone homeostasis, has immunomodulatory properties thought to be beneficial in MS. It is therefore an attractive and fairly safe candidate for add-on therapy. Certain studies also point towards an inverse correlation between serum vitamin D levels and MRI disease activity or relapse rate in MS patients [1, 2].

However, is there a rationale for vitamin D supplementation in MS patients specifically, besides correcting a documented serum deficiency? One can argue that vitamin $\mathrm{D}$ deficiency is also present in a large proportion of the general population in the Northern hemisphere, because of insufficient sunlight exposure, and therefore, supplementation should not be restricted to MS patients only.

The article by Faridar et al. in this issue of Acta Neurologica Belgica provides a critical and comprehensive review of existing preclinical and clinical data, as well as of ongoing trials in this field. This review is particularly timely as several clinical trials have been published in 2012. Although several radiological and clinical parameters have been evaluated, positive outcomes are until now modest or absent. This could be because studies were not adequately powered, the duration of the trial was insufficient or correct supplementation could not be attained in all patients. In addition, certain studies have used different

V. van Pesch $(\square)$. C. J. M. Sindic

Neurology Department, Cliniques Universitaires Saint-Luc,

Brussels, Belgium

e-mail: vincent.vanpesch@uclouvain.be

V. van Pesch - C. J. M. Sindic

Unité de Neurochimie, Institute of Neuroscience,

Université Catholique de Louvain, Brussels, Belgium vitamin $\mathrm{D}$ formulations, making it difficult to draw firm conclusions [3].

Several questions remain therefore unanswered, as to the correct dosage, form and duration of supplementation needed, as well as the ideal target population of MS patients. Furthermore, preventive strategies during childhood and adolescence for high-risk populations (i.e. children of MS patients) have not yet been evaluated. Larger randomized trials are ongoing and will hopefully provide further answers $[4,5]$. In the meantime, supplementation is well-tolerated, safe, at a low cost and should be prescribed in vitamin D-deficient patients. We are, however, still awaiting data that will translate this empirical approach into "evidence-based" therapeutic guidelines applicable in the routine clinical practice.

Conflict of interest None.

\section{References}

1. Runia TF et al (2012) Lower serum vitamin D levels are associated with a higher relapse risk in multiple sclerosis. Neurology 79(3): 261-266

2. Mowry EM et al (2012) Vitamin D status predicts new brain magnetic resonance imaging activity in multiple sclerosis. Ann Neurol 72(2):234-240

3. Stein MS et al (2011) A randomized trial of high-dose vitamin D2 in relapsing-remitting multiple sclerosis. Neurology 77(17): $1611-1618$

4. Dorr J et al (2012) Efficacy of vitamin D supplementation in multiple sclerosis (EVIDIMS Trial): study protocol for a randomized controlled trial. Trials 13:15

5. Smolders J et al (2011) Efficacy of vitamin D3 as add-on therapy in patients with relapsing-remitting multiple sclerosis receiving subcutaneous interferon beta-1a: a Phase II, multicenter, doubleblind, randomized, placebo-controlled trial. J Neurol Sci 311(1-2): $44-49$ 